\title{
Effects and Research of Electric Vehicles Integration Into Power Systems
}

\author{
Li Yan ${ }^{1, a}$, Zhou Yusheng ${ }^{2, b}$
}

(College of Electrical Engineering and Information, Changsha University of Science and Technology, Changsha 410004, China)

a736931631@qq.com, ${ }^{\text {b } z y s d 10193 @ s i n a . c o m, ~}$

\begin{abstract}
Keywords: Electric vehicle power system , Power supply mode, Power quality problem ,Hilbert Huang transform
\end{abstract}

Abstract :Future electric cars (plug-in electric vehicle, PEV) large-scale access, will bring to power system planning and operation can not be ignored.This article first introduces the research status of electric vehicles, including its development scale, technical characteristics and power supply mode, and points out that due to regional differences between the consideration of different factors. Then brainstorm the electric car a lot on the market after use, starting from the power generation side and distribution side, which will bring both the improvements and new problems.Power quality problem for electric cars, is proposed in this paper, based on the Hilbert Huang transform (HHT) classification of power quality disturbance identification method and time of the positioning method.Based on Matlab7.0 simulation platform, because of the influence of the length, only for one of the power quality problems (voltage sag) for signal recognition and time orientation, the simulation results show that the method has good recognition ability and precision.

\section{Introduction}

As electric cars, people also more and more attention to it, it embodies the advantages of energy saving, environment is also very obvious, and it also can promote the application of renewable energy. Literature [1] put forward the thought of V2G earlier and a benefit analysis was carried out. Analyzed the V2G literature [2] to enhance the role of wind capacity and reduce $\mathrm{CO} 2$ emissions. However, a large amount of electric charge and discharge will also bring more problems to the stability of power grid, especially large sensitive power electronic equipment is widely used in electric power system, power quality problems of the research is particularly important.Power quality problem for electric cars, this paper analyzes the power quality disturbance detection based on different methods and the research status of classification recognition, is proposed based on the Hilbert Huang transform (HHT) - the classification of power quality disturbance identification method and time locating method

\section{The Electric Car}

Especially in recent years, the fast development of electric vehicles in China, the state has formulated a series of special preferential policy about electric vehicles, such as tax cuts, no line.Pure electric vehicles as the most environmentally friendly vehicles will replace fuel cars, however, the resulting power quality problems will be more and more prominent.

\section{Development Scale}

Pure electric vehicle research and development of China and abroad was basically the same starting line, technology and industrialization of gap is smaller.Experienced a long-term development, pure electric vehicle technology mature gradually, and in the United States, and other countries for commercial application.Currently has nearly 40000 vehicles in the world of pure electric passenger cars running is mainly used in public transport system.Pure electric cars, although there is no 
China.Europe and the United States and other countries started early, but has incomparable advantages in environmental protection and energy saving, the coming era of new energy vehicles [3].

\section{Technical Characteristics}

In the development of pure electric vehicle research and development, we must resolve and overcome the key technology in four aspects: the battery and battery technology, motor driver and its electronic intelligent control technology, vehicle safety and energy management technology, etc.Batteries and battery is the power source of the pure electric vehicles, is always the important factors which restrict the development of the pure electric vehicle research and development. Now more mature and used batteries for lead-acid batteries, nickel cadmium battery, nickel metal hydride batteries or lithium ion battery [4].At present, mainly by motor of electric vehicle driven by induction motor vector control and direct torque control.

\section{Power Supply Mode}

The electric vehicle power supply way of charging mode and in electric mode. Compared with charging pile, such as charging mode, in the electric model able to occupy less public resources. Compared with the charging mode, electric vehicle power supply in electric mode speed, and can concentrate during low load for battery charging. In charging station can also be combined with a new energy power generation [5] or power system as an important energy storage part [6]. But, in electric mode has such problems as battery interface standard is difficult to unity.

\section{The Influence of Electric Vehicle Access to The Power System}

The use of electric cars to the entire power system will produce influence, from the generation, transmission and distribution side, and the user, will have different levels of change. Power quality problem for electric cars, this paper analyzes the power quality disturbance detection based on different methods and the research status of classification recognition, is proposed based on the Hilbert Huang transform (HHT) - the classification of power quality disturbance identification method and time locating method.

\section{Impact On The Power Generation Side}

When the electric car after a large number of grid, both can be used as a user and can be used as energy storage power. As a user, the electric car can charge during load low concentrated charging the battery or electric cars itself, so you have to eliminate peak valley difference day and night, smooth load effect, reduce the workload of the power generation personnel, enhanced the working economy of the generating set, prolong the service life of engine equipment. Literature [7] put forward under the condition of charging demand constraint electric cars involved in the system frequency regulation control method. As the energy storage power, the electric car is a kind of distributed power supply, has certain controllability, can promote the application of renewable energy. Literature [8] considering the randomness, the electric car car user behavior research electric vehicles to provide the possibility of standby power grid operation.

\section{Impact On The Grid}

Electric vehicle access to the influence of the grid is indirect. As a large number of electric vehicle access, lead to the growth of the power load, and charging load uncertainty will focus on the grid security, economic operation, and the grid planning an impact in the future. Literature [9] to study the electric car access a city in Australia after the grid lines and the influence of the substation main 
equipment, the simulation results show that the electric car of the large-scale access will not lead to the city has a main equipment overload of transmission lines and substations.

\section{The Influence of The Distribution Network}

Electric vehicle access to the power system is mainly in the aspect of power distribution network, the influence of the current main research content involves the power quality, reliability and economic operation of the distribution network, etc., in terms of distribution network and economic operation, the main research of distribution network loss after electric vehicle access and the influence of the distribution transformer life [10]. Key research on the influence of the distribution network power quality.

Access to power quality of distribution network of electric vehicle (ev) mainly effects include voltage drop[11], harmonic pollution[12] and unbalanced three-phase [13]. Therefore need fixing the energy disturbance signal detection classification, current methods mainly include the wavelet transform and $\mathrm{S}$ transform and the text of the Hilbert transform. This paper puts forward for the detection of transient disturbance signal positioning based on Hilbert transform

Hilbert - Huang transform (Hilbert Huang transform, HHT) is Huang in 1998 put forward a new kind of signal analysis theory, it puts forward the Intrinsic Mode Function (the Intrinsic Mode Function, the IMF) and introduce the method of Empirical Mode Decomposition (Empirical Mode Decomposition, the EMD), very suitable for analyzing real life common non-linear and non-stationary signal frequency changes over time in [14] .

\section{Power Disturbance Signal Classification}

Disturbance signal classification is an important content in signal recognition, to identify the actual waveform of power quality disturbance is a signal classification problem, the key is according to the transform method to get the characteristics of it, choose a better decision-making function, and establish the indicators reflect the good and bad level of the decision function, decision-making function make the signal classification problem pay more attention to the action of actual effect [15].At present, based on the signal classification method mainly include artificial neural network, bayesian classification, expert system, support vector machine (SVM) [16]. And by the Hilbert Huang transform the EMD of the decomposed each IMF component is smooth, as you progress through the decomposition, the IMF component in frequency components reduce gradually. As a result, the signal in different scale fluctuations and trends come from high to low down step by step, and thus to identify the characteristics of the signal, as soon as possible to determine the type of power quality disturbance, has the good local adaptive .

The EMD of the specific steps are: to a real signal $x(t)$, first identify $x(t)$ all of the maximum points, then all the maximum points with cubic spline curve interpolation signal $x(t)$ envelope curve $e_{\max }(t)$. Similarly, can get the signal $x(t)$ envelope curve $e_{\min }(t)$.

The envelope curve $e_{\max }(t)$ and the envelope curve $e_{\min }(t)$ on the average, the mean envelope curve $m_{1}(t)$

$$
m_{1}(t)=e_{\max }(t)+e_{\min }(t)
$$

Use $x(t)$ subtract to $m_{1}(t)$ get the first component, namely:

$$
x(t)-m_{1}(t)=h_{1}(t)
$$




\section{Power Disturbance Signal Threshold Extraction}

Pulse extraction algorithm based on adaptive threshold makes threshold, along with the change of background noise and interference pulse level changes and adaptive. Based on the literature [17], for the data length is $\mathrm{N}$ sampling signal $\mathrm{x}(\mathrm{k})$, the threshold lambda can be calculated by the next type:

$$
\sigma \approx \delta=\sqrt{\frac{1}{N} \sum_{\mathrm{n}-1}^{N} d_{1}^{2}}(n)
$$

In the formula , $\mathrm{N}$ for the length of the data. When $\mathrm{d}_{1}(\mathrm{n})$ in the pulse signal, the sigma value increase, lambda also increase, therefore the threshold has change with background noise, pulse level and ability of adaptive adjustment.But this kind of method is only applicable to the high frequency signal, and threshold calculation is quite trouble.

A large number of simulation results show that, by empirical mode decomposition method after the signal is decomposed into a finite number of IMF function, the IMF1 after the Hilbert transform, only in the event of a disturbance moment, IMF derivation after amplitude will have a lot of mutations, but no disturbance occurs in a point in time, amplitude change is not big, after the IMF derivative. Therefore, better determine this topic threshold. The Hilbert spectrum analysis of Hilbert Huang transform. It is the basic content of the Hilbert transform of each IMF function and to solve the instantaneous frequency can get time-frequency energy distribution in the plane of the signal, namely the Hilbert spectrum.

For any continuous time signal $x(t)$, The Hilbert transform definitions $\hat{x}(t)$ as :

$$
\hat{x}(t)=\frac{1}{\pi} \int_{-\infty}^{+\infty} \frac{x(u)}{t-u} d u
$$

The inverse transform is:

$$
x(t)=\frac{1}{\pi} \int_{-\infty}^{+\infty} \frac{\hat{x}(u)}{u-t} d u
$$

\section{Power Disturbance Signal Time Orientation}

Based on Matlab7.0 simulation platform, the simulation and analysis of the common several kinds of transient power quality disturbance, after complete the Hilbert - Huang transform, get the Hilbert spectrum, extract its characteristic parameters, and carries on the location and time study. Table 1 to introduce electric vehicles produce several kinds of common power quality disturbance signals

Table 1. Power Quality Disturbances Model and Class Symbol

\begin{tabular}{lcc} 
The disturbance type & The signal model & Parameter Settings \\
\hline Sine signal & $x(t)=A \sin (\omega t)$ & $A=1(p . u),. f=50 H z$ \\
\hline Voltage sag & $x(t)=A\left\{1-\alpha\left[u\left(t_{2}\right)-u\left(t_{1}\right)\right]\right\} \sin (\omega t)$ & $0.1 \leq \alpha \leq 0.9, T \leq t_{2}-t_{1} \leq 15 T$ \\
\hline & $x(t)=A\left[\alpha_{1} \sin (\omega t)+\alpha_{3} \sin (3 \omega t)+\right.$ & $0.05 \leq \alpha_{3} \leq 0.15,0.05 \leq \alpha_{5} \leq 0.15$, \\
& $\left.\alpha_{3} \sin (5 \omega t)+\alpha_{7} \sin (7 \omega t)\right]$ & $0.01 \leq \alpha_{7} \leq 0.08, \sum \alpha_{i}^{2}=1$ \\
Harmonic &
\end{tabular}




\section{Case Analysis}

The use of the Hilbert Huang transform to voltage sag as an example, the first failure area is obtained by empirical mode decomposition method of multi-layer experience modal function graphic, Hilbert transform was carried out on the empirical mode function again, get the Hilbert spectrum after the transformation, to extract the characteristic parameters, then the failure region decomposition and time orientation. With voltage of transient power quality disturbance signal will, for example, signal source formula is as follows:

$$
x(t)=A\left\{1-\alpha\left[u\left(t_{2}\right)-u\left(t_{1}\right)\right]\right\} \sin (\omega t)
$$

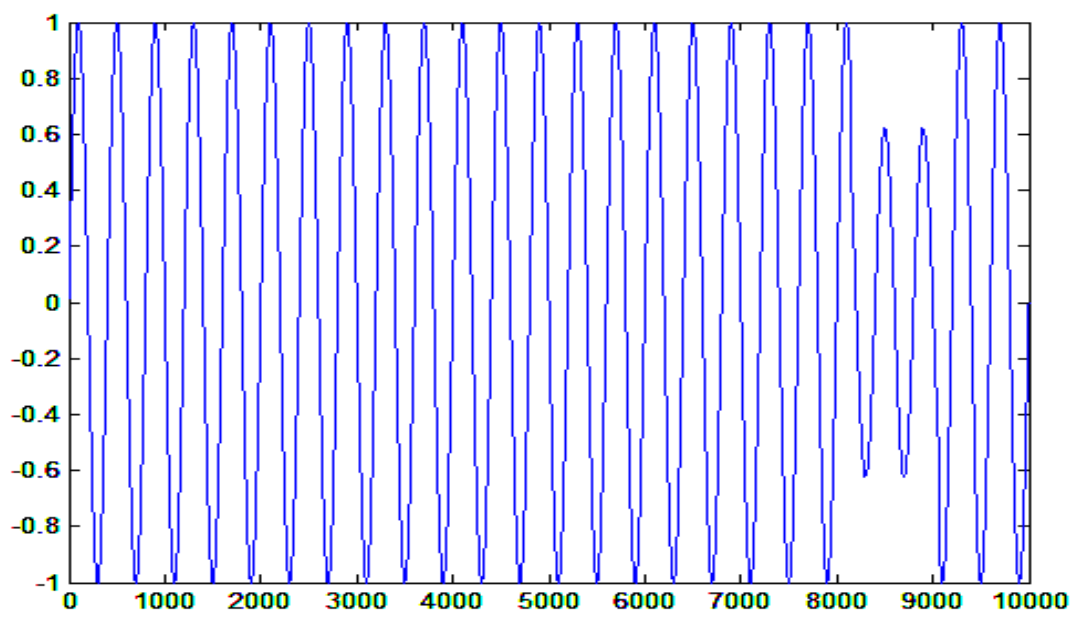

Fig. 1 Voltage Sag Signal

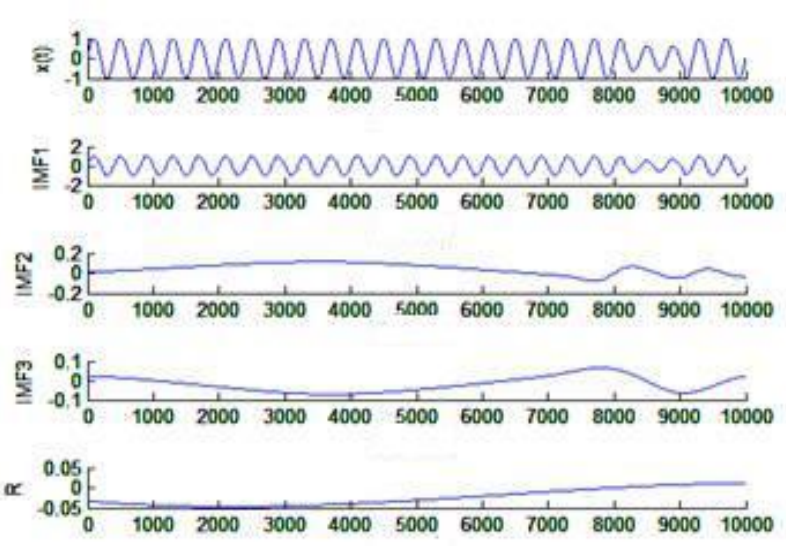

Fig. 2 The IMF Each Component

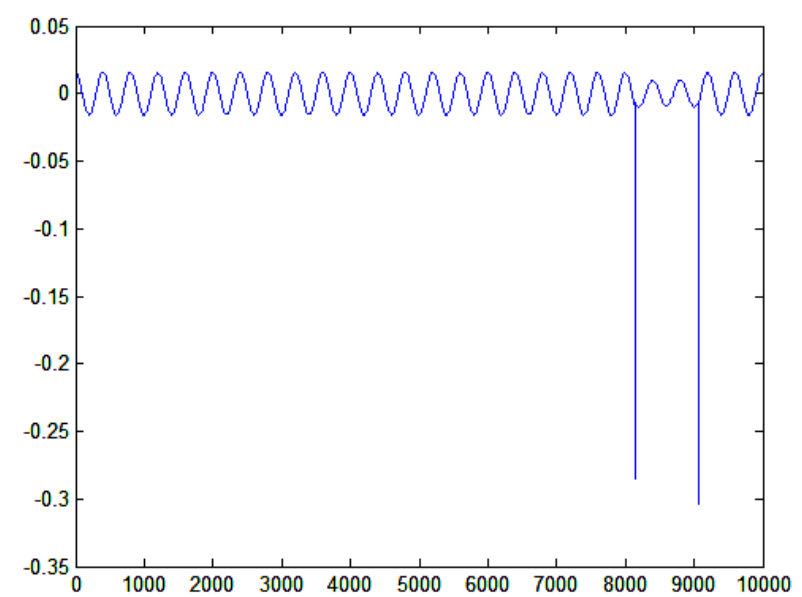

Fig. 3 IMF1 After Hilbert Transform of Graphics

The $\mathrm{X}$ axis for the sampling points, sampling points, a total of $10000, \quad \mathrm{Y}$ for voltage amplitude..In Fig. 2 to Fig. 1 contains the voltage sag of local amplification of signals and by the EMD decomposition of multilayer IMF components.Can be seen from the figure in each layer of the IMF, the result is the real existence of different scales will signal fluctuations or trend from high to low down step by step, so as soon as possible to analyze the characteristics of the signal, has the good local adaptation. Fig. 3 for the IMF in Fig. 2 after the derivation of graphics, abscissa for sampling points, Can be seen from the above, only happened is happened in the time of onset and termination of 
the disturbance amplitude of huge mutation, voltage waveform normal period, amplitude changes after derivation is not big, therefore, to determine the threshold.

Table. 2 Results analysis

\begin{tabular}{|l|l|l|}
\hline & The starting point & $\begin{array}{l}\text { Termination of the } \\
\text { moment }\end{array}$ \\
\hline The actual value & 0.40730 & 0.45289 \\
\hline Measured value & 0.40730 & 0.45280 \\
\hline
\end{tabular}

From the data results are shown in Table 2, the time error is less than $0.02 \%$ 。

\section{Conclusion}

Bold ideas in this paper the electric car a lot on the market after use, due to the charging time not sure, that generates power grid transient power quality disturbance, endanger the stability of power grid. Extracted from signal classification, the threshold, the signal time positioning in three aspects, and combined with the use of the Hilbert Huang transform to classify all kinds of transient disturbance signal power identification and positioning time, and in the case of the simulation verify its validity. The system for the electric charging system for grid power quality problem provides a reference method, have certain forward-looking and operability, has important guiding significance to the development of electric charge system.

\section{Author's brief introduction}

Li Yan(1988-),Female, master graduate student, the study of effects and eesearch of electric vehicles integration into power systems.

E-mail:736931631@qq.com

Zhou Yusheng(1965 -), male, professor, doctor, graduate student teacher, be engaged in transmission line safety and disaster prevention, high temperature superconducting power technology research.

E-mail:zysd10193@sina.com

\section{Reference}

[1] Tomic J, Kempton W .Using fleets of electric-drive vehicles for grid support[J]. Journal of Power Sources,2007. 1 fiR(21 \} 459-4fiR.

[2] Lunda $\mathrm{H}$, Kempton W. Integration of renewable energy into the transport and electricity sectors through V2G[J]. Energy Policy, 2008, 36(9): 3578-3587.

[3] Jian Ouyang, Di Li, LiuJunc Cheng. Electric vehicle power battery charged state estimation method is used to study review [J]. Mechanical and electrical engineering. 2016 (01) (in Chinese).

[4] Shaoyun Ge, Henghui Lian, hong Liu, Jianyi Guo,Jun Han,Hu Li. Electric vehicle temporal response capacity model and control strategy [J]. Automation of electric power systems, 2016 (03) (in Chinese). 
[5] Takagi M, Iwafune Y, Yamamoto H, et al. Energy storage of PV using batteries of battery-switch stations[C]//IEEE International Symposium on Industrial Electronics.Austin: IEEE, 2010: 3413-3419.

[6] Lombardi P, Heuer M, Styczynski Z. Battery switch station as storage system in an autonomous power system:optimization issue[C]//IEEE Power and Energy Society General Meeting. Minneapolis: IEEE, 2010: 1-6.

[7] Fernandez L P, Roman T G S, Cossent R. Assessment of the impact of plug-in electric vehicles on distribution networks[J]. IEEE Trans. on Power System, 2011，26(1):

206-213.

[8] Dallinger D, Krampe D, Wietschel M. Vehicle-to-grid regulation reserves based on a dynamic simulation of mobility behavior[J]. IEEE Trans. on Smart Grid, 2011, 2(2) : 302-313.

[9] Carthy D M, Wolfs P. The HV system impacts of large scale electric vehicle deployments in a metropolitan area[C]//20th Australasian Universities Power Engineering Conference. Christchurch: IEEE. 2010: 1-6.

[10] Soares F J, Lopes JA P, Almeida P M R. A Monte Carlo method to evaluate electric vehicles impacts in distribution networks[C]//IEEE Conference on Innovative Technologies for an Efficient and Reliable Electricity Supply. Waltham: IEEE, 2010: 365-372.

[11] Kelly L, Rowe A, Wild P. Analyzing the impacts of plug-in electric vehicles on distribution networks in British Columbia[C]//Electrical Power Energy Conference.Montreal : IEEE. 2009: $1-6$.

[12] Richardson P, Flynn D, Keane A. Impact assessment of varying penetrations of electric vehicles on low voltage distribution systems[C]//IEEE Power and Energy Society General Meeting. Minneapolis: IEEE, 2010: 1-6.

[13] Sortomme E, Hindi M M, Macpherson S D J, et al.Coordinated charging of plug-in hybrid electric vehicles to minimize distribution system losses[J].IEEE Trans. On Smart Grid, 2011, 2(1): 198-205.

[14] Dugan R C, Megranghan M F, Benty H W. Electrical Power System Quality[M].New York: McGraw-Hill,1996.

[15] Huang N E. The Empirical Mode Decomposition and the Hilbert Spectrum for Nonlinear and Non-stationary Time Series Analysis. J. Proc. R. Soc. Lond. A. 1998， 454:903-995.

[16] Huang N E, Long S R.A new view of nonlinear water waves: The Hilbert Spectrum. Annu. Rev. Fluid. 1999, 5(31):417-457.

[17] Ren Lijia, Sheng Gehao, Zhuang Qikai, et al. Application of ICA on transmission tower theftroof monitoring system[J]. Automation of Electric Power Systems, 2008, 32(2): 97-100(in Chinese). 